\title{
ASYMPTOTIC THEORY OF ESTIMATES OF KTH-ORDER SPECTRA*
}

\section{By D. R. Brillinger and M. Rosenblatt $\dagger$}

LONDON SCHOOL OF ECONOMICS, UNIVERSITY OF CALIFN?NIA, SAN DIEGO (LA JOLLA), AND UNIVERSITY COLLEGE, LONDON

Communicated by John W. Trukey, December 5, 1966

1. Notation and Assumptions.-Let $X(t)$ be a strictly stationary $r$-vector valued process with real-valued components. All moments are assumed to exist. While $t$ may be discrete or continuous, principal results are stated only in the discrete case ( $t$ running through the integers) though the obvious parallels in the continuous case are valid, provided $X(t)$ is assumed to be continuous in mean square.

Existence of second-order moments implies that $X(t)$ has a vector-valued Fourier representation

$$
X(t)=\int e^{i t \lambda} d Z(\lambda)
$$

in mean square with $Z(\lambda)$ an $r$-vector valued process with orthogonal increments. Let $c(Z)=c\left(Z_{1}, \ldots, Z_{k}\right)$ denote the $k$ th-order cumulant of $Z=\left(Z_{1}, \ldots, Z_{k}\right)$, and for $a=\left(a_{1}, \ldots, a_{k}\right), t=\left(t_{1}, \ldots, t_{k}\right)$ let

$$
c_{k, a}(t)=c_{a_{1}}, \ldots, a_{k}\left(t_{1}, \ldots, t_{k}\right)=c_{a_{1}}, \ldots, a_{k}\left(\tau+t_{1}, \ldots, \tau+t_{k}\right)=c\left(X_{a}(t)\right)
$$

be the $k$ th-order cumulant of $X_{a}(t)=\left(X_{a_{1}}\left(t_{1}\right), \ldots, X_{a_{k}}\left(t_{k}\right)\right)$. Using stationarity, write its asymmetric form as

$$
c_{k, a}^{\prime}\left(t^{\prime}\right)=c_{a_{1}, \ldots, a_{k}}\left(\tau+t_{1}, \ldots, \tau+t_{k-1}, \tau\right),
$$

where $t^{\prime}=\left(t_{1}, t_{2}, \ldots, t_{k-1}\right)$.

Assumption I. For each $j=1, \ldots, k-1$ and any $k$-tuple $a_{1}, \ldots, a_{k}$ let

$$
\sum_{t^{\prime}}\left|t_{j} c_{k, a}^{\prime}\left(t^{\prime}\right)\right|<\infty
$$

where $k=2,3, \ldots$

Assumption I implies that all cumulant spectral densities

$$
\begin{aligned}
& f_{k, a}^{\prime}\left(\omega^{\prime}\right)=f^{\prime}{ }_{a_{1}}, \ldots, a_{k}\left(\omega_{1}, \ldots, \omega_{k-1}\right)=f_{k, a}(\omega)=f_{a_{1}}, \ldots, a_{k}\left(\omega_{1}, \ldots, \omega_{k}\right) \\
& =(2 \pi)^{-k+1} \sum_{t^{\prime}} c_{k, a}^{\prime}\left(t^{\prime}\right) \exp \left(-i \sum_{j=1}^{k-1} t_{j} \omega_{j}\right)
\end{aligned}
$$

exist where it is understood that

$$
\sum_{j=1}^{k} \omega_{j} \equiv 0 \bmod 2 \pi \quad j=1, \ldots, k
$$

in formula (1.5). Further, Assumption I implies that all the cumulant spectral densities are continuous and continuously differentiable. The cumulant

$$
c\left(d Z_{a}(\omega)\right)=f_{k, a}(\omega) \eta\left(\sum_{1}^{k} \omega_{j}\right) d \omega
$$

where $\eta(x)=\sum_{-\infty}^{\infty} \delta(x+2 j \pi)$ with $\delta(x)$ the Dirac delta function and $d \omega=\prod_{1}^{k} d \omega_{j}$. 
Let

$$
d_{a_{j}}^{(T)}\left(\lambda_{j}\right)=\sum_{t=0}^{T-1} X_{a_{j}}(t) \exp \left(-i \lambda_{j} t\right)
$$

and

$$
I_{k, a}{ }^{(T)}(\lambda)=(2 \pi)^{-k+1} T^{-1} \prod_{j=1}^{k} d_{a_{j}}^{(T)}\left(\lambda_{j}\right)
$$

with $\sum_{j=1}^{k} \lambda_{j} \equiv 0 \bmod 2 \pi$. The function $I_{k, a}^{(T)}(\lambda)$ is a $k$ th-order analogue of the second-order periodogram and cross-periodogram. The following lemma is useful in analyzing the asymptotic behavior of a $k$ th-order periodogram and estimates of $k$ th-order spectra based on the periodogram.

Lemma. Suppose Assumption I is satisfied. Then the cumulant

$$
\begin{aligned}
c\left(d_{a_{1}}{ }^{(T)}\left(\lambda_{1}\right), \ldots d_{a_{k}}{ }^{(T)}\left(\lambda_{k}\right)\right) & \\
& =(2 \pi)^{-k+1} f^{\prime}{ }_{k, a}\left(\lambda^{\prime}\right) \sum_{t=0}^{T-1} \exp \left(-i \sum_{1}^{k} \lambda_{j}\right)+0(1),
\end{aligned}
$$

where the error term $0(1)$ is uniform in $\lambda_{1}, \ldots, \lambda_{k}$ as $T \rightarrow \infty$.

2. A Class of Estimates.- Let $W(u)$ be a bounded continuous weight function on the plane $\sum_{1}^{k} u_{j}=0$ symmetric about zero, $W(u)=W(-u)$ with

$$
\int W(u) \delta\left(\sum_{1}^{k} u_{j}\right) d u=1 .
$$

Assumption II. Let $W(u)$ be continuously differentiable with

$$
\left|u_{j} W(u)\right|,\left|\frac{\partial}{\partial u_{j}} W(u)\right| \leq M(1+\|u\|)^{-k+1-~},
$$

$\|u\|=\left(\sum_{1}^{k} u_{j}^{2}\right)^{1 / 2}$, uniformly in $j$ and $u=\left(u_{1}, \ldots, u_{k}\right)$ where $M, \epsilon>0$. Set

$$
W_{T}(u)=B_{T}^{-k+1} \Sigma^{\prime} W\left(B_{T}^{-1}(u+2 \pi j)\right),
$$

where the summation is over $j=\left(j_{1}, \ldots, j_{k}\right)$ such that $\sum_{1}^{k}\left(j_{\alpha}+u_{\alpha}\right)=0$ and $B_{\underline{q}}$ is chosen so that $B_{T} \rightarrow 0$ as $T \rightarrow \infty$ but ${B_{T}}^{k-1} T \rightarrow \infty$ as $\dot{T} \rightarrow \infty$.

The estimates of $f_{k, a}(\lambda), \sum_{1}^{k} \lambda_{j} \equiv 0 \bmod 2 \pi$, that we consider take the form

$$
\begin{aligned}
f_{k, a}{ }^{(T)}(\lambda)=\left(\frac{2 \pi}{T}\right)^{k-1} \sum_{s_{1}, \ldots, s_{k}=0}^{T-1} W_{T}\left(\lambda_{1}-\frac{2 \pi s_{1}}{T}, \ldots, \lambda_{k}-\frac{2 \pi s_{k}}{T}\right) \\
\times \Phi\left(\frac{2 \pi s_{1}}{T}, \ldots, \frac{2 \pi s_{k}}{T}\right) I_{k, a}(T)\left(\frac{2 \pi s_{1}}{T}, \ldots, \frac{2 \pi s_{k}}{T}\right),
\end{aligned}
$$

where $\Phi(u)$ is zero unless $u$ is on the manifold $\sum_{1}^{k} u_{j} \equiv 0 \bmod 2 \pi$, but not on a 
proper submanifold $\sum_{j \in I} u_{i} \equiv 0 \bmod 2 \pi$ with $I$ a proper nonvacuous subset of $(1, \ldots, k)$, in which case it is one.

THEOREM 1. Let $X(t)$ be an r-vector valued strictly stationai'y process satisfying Assumption I. Let $f_{k, a}{ }^{(T)}(\lambda)$ be an estimate of $f_{k, a}(\lambda)$ of the type given in (2.4) with weight function $W$ satisfying Assumption II. If $B_{T} T \rightarrow \infty$ as $B_{T} \rightarrow 0$ and $T \rightarrow \infty$, then

$$
E f_{k, a}{ }^{(T)}(\lambda)=f_{k, a}(\lambda)+0\left(B_{T}\right)+0\left(B_{T^{-1}} T^{-1}\right)
$$

Thus we have a class of asymptotically unbiased estimates under the assumption made in Theorem 1. The following results describe the asymptotic behavior of the covariance of two such estimates. It is remarkable that to the first order the asymptotic behavior of covariances of $k$ th-order cumulant spectral density estimates depends only on second-order spectra under the assumptions made. The covariance of two complex-valued random variables $X, Y$ is taken to be $\operatorname{cov}(X, Y)$ $=E X \bar{Y}-(E X)(E \bar{Y})$.

Theorem 2. Let $X(t)$ be an r-vector valued strictly statiomary process satisfying Assumptiom I. Let $f_{k, a}{ }^{(T)}(\lambda)$ and $f_{k, b}{ }^{(T)}(\mu)$ be estimates of $f_{k, a}(\lambda)$ and $f_{k, b}(\mu)$, respectively, of the type given in formula (2.4) with weight function $W_{T}$ satisfying Assumption II. Then

$$
\begin{array}{r}
\operatorname{cov}\left[f_{k, a}{ }^{(T)}(\lambda), f_{k, b}{ }^{(T)}(\mu)\right]=2 \pi T^{-1} \sum_{P} \int_{j} W_{T}(\lambda-\alpha) W_{T}(\mu-\beta) \eta\left(\sum_{1}^{k} \alpha_{j}\right) \\
\left.\times \underset{1}{k} \eta\left(\alpha_{j}-(P \beta)_{j}\right) f^{\prime}{ }_{a_{j},(P b)_{j}}\left(\alpha_{j}\right)\right) d \alpha d \beta+0\left(B_{T^{-k+2}} T^{-1}\right),
\end{array}
$$

where the summation is over all permutations $P$ on the integers $1, \ldots, k\left(P b=\left(b_{P 1}\right.\right.$, $\left.\ldots, b_{P k}\right)$ ), and the error term is uniform in $\lambda$ and $\mu$ subject to $\sum_{1}^{k} \lambda_{j}, \sum_{1}^{k} \mu_{j} \equiv 0 \quad \bmod 2 \pi$.

Corollary. Under the assumptions of Theorem 2,

$$
\begin{aligned}
\lim _{T \rightarrow \infty} B_{T}{ }^{k-1} T \operatorname{cov}\left[f_{k, a}{ }^{(T)}(\lambda), f_{h, b}{ }^{(T)}(\mu)\right]=2 \pi \sum_{P} & \stackrel{(\Pi=1}{k}\left\{\left\{\lambda_{j}-(P \mu)_{j}\right\} f^{\prime}{ }_{a_{j},(P b) j}\left(\lambda_{j}\right)\right) \\
& \int W(\beta) W(P \beta) \delta\left(\sum_{1}^{k} \beta_{j}\right) d \beta,
\end{aligned}
$$

where the summation is over all permutations $P$ on the integers $1, \ldots, k$. Here $\eta\{\lambda\}=$ $\sum_{-\infty}^{\infty} \delta\{\lambda+2 j \pi\}$ with $\delta\{\lambda\}$ the Kronecker delta.

Consider estimates of cumulant spectra of orders $k_{1} \leq k_{2} \leq \ldots \leq k_{m}$ of the form given in (2.4) with scale factors $B_{T}{ }^{(1)} \leq \ldots \leq B_{T}{ }^{(m)}$. Write the $j$ th such estimate in the form

$$
f_{A j}{ }^{(T)}\left(\lambda^{(j)}\right)=\left(\frac{2 \pi}{T}\right)^{k_{j}-1} \sum W_{T}{ }^{(j)}\left(\lambda^{(j)}-\frac{2 \pi s^{(j)}}{T}\right) \Phi\left(\frac{2 \pi s^{(j)}}{T}\right) I_{A j}{ }^{(T)}\left(\frac{2 \pi s^{(j)}}{T}\right)
$$

where $A_{j}$ denotes the indices of the $k_{j}$ series involved in the $j$ th estimate. The scale factors of two estimates of the same order will be taken to be the same.

TheOREm 3. Let $X(t)$ be an r-vector valued strictly statiomary process satisfying As- 
sumption I. Let $f_{\mathrm{A}_{j}}{ }^{\left({ }^{\prime}\right)}\left(\lambda^{(j)}\right), j=1, \ldots, m$, be estimates as given by (2.8) whose weight functions $W_{T^{(j)}}^{(j a t i s f y ~ A s s u m p t i o n ~ I I . ~ T h e n ~ t h e ~ e s t i m a t e s ~ a r e ~ a s y m p t o t i c a l l y ~ j o i n t l y ~}$ normally distributed as $T \rightarrow \infty$ with estimates of different orders asymptotically independent and estimates of the same order having covariance structure given by (2.7).

The results given above are for discrete parameier processes. Using the analogue of Assumption I for a continuous parameter process and Assumption II, parallel results for a continuous parameter process continuous in the mean are valid, with $\eta$ in formulas (2.8) and (2.9) replaced by $\delta$.

3. Aliasing.-Often a continuous time parameter strictly stationary process $X(t)$ is sampled discretely at time points $j h, j=0, \pm 1, \ldots$, where $h>0$. The analogue of Assumption I for a continuous time parameter process implies that the corresponding cumulant spectral densities $g_{h, a}(\lambda), \sum_{1}^{k} \lambda_{\alpha}=0,-\infty<\lambda_{\alpha}<\infty$, exist, are continuous and continuously differentiable. In addition, we will require

Assumption III. Let

$$
\left|g_{k, a}(\lambda)\right| \leq M(1+\|\lambda\|)^{-k+1-\epsilon}
$$

and

$$
\left|c_{k, a}^{\prime}\left(t^{\prime}\right)\right| \leq M\left(1+\left\|t^{\prime}\right\|\right)^{-k+1-\epsilon}
$$

uniformly in $\lambda$ and $t^{\prime}$ with $M, \epsilon>0$.

Under Assumption III the cumulants for the continuous time parameter process $X(t)$ have the following Fourier representation in terms of the cumulant spectral densities

$$
c_{k, a}(\dot{t})=\int \exp \left(i \sum_{j=1}^{k} t_{j} \lambda_{j}\right) g_{k, a}(\lambda) \delta\left(\sum_{1}^{k} \lambda_{j}\right) d \lambda .
$$

The discretely sampled process $X(j h)$ has cumulants with the corresponding Fourier representation

$C_{a_{1}, \ldots, a_{k}}\left(j_{1} h, \ldots, j_{k} h\right)$

$$
\begin{aligned}
=\int \exp \left(i \sum_{\alpha=1}^{k} j_{\alpha} h \lambda_{\alpha}\right) \delta\left(\sum_{1}^{k} \lambda_{j}\right) g_{k, a}(\lambda) d \lambda \\
=\int_{-\pi / h}^{\pi / h} \int \exp \left(i \sum_{\alpha=1}^{k} j_{\alpha} \lambda_{\alpha} h\right) \eta\left(\sum_{\alpha=1}^{k} h \lambda_{\alpha}\right) f_{k, a}(\lambda) d \lambda
\end{aligned}
$$

in terms of the corresponding cumulant spectral density of $X(j h)$

$$
f_{k, a}(\lambda)=\sum^{\prime} g_{k, a}\left(\lambda+\frac{2 \pi j}{h}\right)
$$

where the sum in (3.5) is over $j=\left(j_{1}, \ldots, j_{k}\right)$ such that $\frac{k}{\sum_{1}} j_{\varkappa}=-(h / 2 \pi) \sum^{k} \lambda_{\alpha}$.

4. Previous Work.-Moments of order $k$ of the $d Z_{a}(\omega)$ have been considered by Blanc-Lapierre and Fortet. ${ }^{1}$ The third-order spectral density, or bispectrum, is defined in Tukey, ${ }^{2}$ and asymptotic properties of a class of estimates are given in Rosenblatt and Van Ness. ${ }^{3}$ Asymptotic properties of a class of estimates of the $k$ th-order cumulant spectra have been considered in Brillinger. ${ }^{4}$ 
Summary.-Under appropriate assumptions the asymptotic variance and bias of a class of estimates of the $k$ th-order cumulant spectra of a stationary random process are obtained. The estimates are shown to be distributed asymptotically as complex-valued Gaussian variables. Remarks are made on aliasing.

* Research partially supported by Office of Naval Research.

† John Simon Guggenheim Fellow, 1965-1966.

${ }^{1}$ Blanc-Lapierre, A., and R. Fortet, Théorie des Fonctions Aléatoires (Paris: Masson, 1953).

2 Tukey, J. W., "An introduction to the measurement of spectra," in Probability and Statistics, ed. U. Grenander (New York: Wiley, 1959), the Harald Cramér volume.

${ }^{3}$ Rosenblatt, M., and J. W. Van Ness, "Estimation of the bispectrum," Ann. Math Statist., $36,1120-1136$ (1965).

"Brillinger, D. R., "An introduction to polyspectra," Ann. Math. Statist., 36, 1351-1374 (1965). 